\title{
INFORMATION-THEORETIC ANALYSIS OF STOCHASTIC VOLATILITY MODELS
}

\author{
OLIVER PFANTE* and NILS BERTSCHINGER ${ }^{\dagger}$ \\ Frankfurt Institute for Advanced Studies, \\ Systemic Risk Group, Frankfurt a. Main, \\ Hesse 60438, Germany \\ *pfante@fias.uni-frankfurt.de \\ †bertschinger@fias.uni-frankfurt.de \\ Received 19 February 2018 \\ Revised 29 August 2018 \\ Accepted 2 November 2018 \\ Published 10 January 2019
}

\begin{abstract}
Stochastic volatility models describe asset prices $S_{t}$ as driven by an unobserved process capturing the random dynamics of volatility $\sigma_{t}$. We quantify how much information about $\sigma_{t}$ can be inferred from asset prices $S_{t}$ in terms of Shannon's mutual information in a twofold way: theoretically, by means of a thorough study of Heston's model; from a machine learning perspective, by means of investigating a family of exponential Ornstein-Uhlenbeck (OU) processes fitted on S\&P 500 data.
\end{abstract}

Keywords: Information theory; stochastic volatility; Bayesian analysis.

\section{Introduction}

Many plaudits have been aptly used to describe Black and Scholes' [4] contribution to option pricing theory. However, especially after the $1987 \mathrm{crash}$, the geometric Brownian motion model and the Black-Scholes formula were unable to reproduce the option price data of real markets. This is not surprising because the Black-Scholes model makes the strong assumption that log-returns of stocks are normally and independently distributed with a volatility which is not only assumed to be known but also constant over time. Both assumptions on volatility are wrong: first, volatility is a hidden parameter which needs to be inferred from stock and option data, respectively; second, volatility is not constant at all but a highly volatile time process. Among the most relevant statistical properties, volatility seems to be

\footnotetext{
${ }^{*}$ Corresponding author.
}

This is an Open Access article published by World Scientific Publishing Company. It is distributed under the terms of the Creative Commons Attribution 4.0 (CC-BY) License. Further distribution of this work is permitted, provided the original work is properly cited. 
responsible for the observed clustering in price changes. That is, large fluctuations are commonly followed by other large fluctuations and similarly for small changes [7]. Another feature is that in clear contrast with price changes which show negligible autocorrelations, volatility autocorrelation is still significant for time lags longer than one year [7, 14, 19, 20, 26, 24]. Additionally, there exists the so-called leverage effect, i.e., much shorter (few weeks) negative cross-correlation between current price change and future volatility [4-7].

Inspired by these observations, stochastic volatility models have been developed which describe time-varying volatility as well as dependencies among stock returns. Here, we build on Heston's model [17] and the exponential Ornstein-Uhlenbeck (OU) model $[2,23,26]$. We select Heston's model for two reasons: empirically, it reproduces several important stylized facts of daily price data, namely leverage effect, timevarying volatility and fat tails $[10,12,22,30]$; theoretically, there is an explicit formula for the characteristic function of the asset log-price [12, 17]. Furthermore, these results allow to efficiently compute option prices under the Heston model $[12,30]$ which is routinely calibrated in option trading. While building on some of these analytic results, we do not consider option pricing in the present paper. Instead, we pose the following question: how reliably can the hidden volatility be inferred from observable stock return data alone? Since, in stochastic volatility models stock prices as well as the time varying volatility are both modeled as random variables, Shannon's information theory [32] provides an ideal frame to make this question precise: how much information do observed stock returns provide about the hidden volatility? To our knowledge, this point has not been discussed in the literature, let alone been studied in a rigorous and quantitative fashion.

Thanks to the analytic tractability of the considered stochastic volatility models we can not only quantify the information content of a single stock price observation about the underlying volatility, but also quantify to which extent the stock process can be considered as a self-contained process: a notion which was made precise by Pfante et al. [27] as outlined in Sec. 2.3. However, if we want to incorporate more than one asset price observation, the analytical approach for Heston's model becomes infeasible and we treat this problem in the realm of exponential OU single- and twofactor models. Masoliver and Perello [23] suggested such models which are of similar analytic tractability [26] as Heston's model. Furthermore, two-factor models are often considered necessary to fit empirical return data [9] as well as volatility surfaces [25]. In exponential OU models, the logarithm of stochastic volatility follows a Gaussian process. We generalize the class of exponential OU models even further by considering arbitrary Gaussian processes driving the logarithm of the volatility. This allows us to utilize powerful tools from machine learning to fit such models to stock price data and compare their relative performance. We can replicate some of the findings regarding stylized facts on volatility dynamics, e.g., long-range correlations and clustering. Most importantly, building on information theoretic results for Gaussian distributions we can precisely estimate the information content of stock prices in these models. 
Overall, our results are quite disillusioning. Stock prices provide only limited information about the volatility: the information gain from observing long stock price trajectories is only about a single bit, and it quickly saturates, that is, only very recent stock price movements over the previous 20 days provide any valuable information about current volatility. This general finding concurs with a theoretical observation we make for the Heston model: the stock process is a nearly self-contained Markovian process which can be described in its own terms without referring to its underlying volatility.

Our paper is structured as follows: first, we introduce stochastic volatility models in general, Heston's model and exponential OU models in particular. For the first one, we present the analytic solutions for the transition probabilities. Then, we provide a brief recapitulation of information theory and put the results by Pfante et al. [27] on multilevel dynamical systems into the scope of the present paper. After assembling all these prerequisites we present the results: first, for the Heston model we numerically compute the information content between stocks and volatility and the information measure which quantifies to which extent the stock process in Heston's model is self-contained; second, the results for the exponential OU models. We then conclude by discussing our findings.

\section{Prerequisites}

\subsection{Stochastic volatility models}

As in most stochastic volatility models, we consider a stock, whose price $t \rightarrow S_{t}$ process follows a geometric Brownian motion:

$$
d S_{t}=\mu S_{t} d t+\sigma_{t} S_{t} d W_{t}^{(1)}
$$

with a fixed initial value $S_{0} . \mu$ is the drift parameter, $W_{t}^{(1)}$ a standard Brownian motion, $\sigma_{t}$ the time dependent volatility. Since the geometric Brownian motion depends on $\sigma_{t}^{2}$ we introduce the variance $v_{t}=\sigma_{t}^{2}$ described by the stochastic differential equation (SDE)

$$
d v_{t}=\mu\left(v_{t}\right) d t+\beta\left(v_{t}\right) d W_{t}^{(2)},
$$

where the functions $\mu$ and $\beta$ are assumed to be smooth, and $\beta(v)>0$ for all $v$. In the sequel we shall replace the generic functions $\mu$ and $\beta$ by more specific ones if we consider the Heston model or the exponential OU models. We allow for a coupling between the two Brownian motions, that is, $d W_{t}^{(2)}=\rho d W_{t}^{(1)}+\sqrt{1-\rho^{2}} d Z_{t}$ where $Z_{t}$ is a Brownian motion independent of $W^{(1)}$ such that $\mathbb{E}\left[d W_{t}^{(1)} d W_{t}^{(2)}\right]=\rho d t$.

\subsubsection{Heston model}

In Heston's model [17] the time evolution $t \rightarrow v_{t}$ of the variance follows the Cox-Ingersoll-Ross (CIR) process:

$$
d v_{t}=-\gamma\left(v_{t}-\theta\right) d t+\kappa \sqrt{v_{t}} d W_{t}^{(2)}
$$


where $\theta$ is the long-term mean of $v_{t}, \gamma>0$ the rate of relaxation to this mean, $\kappa>0$ the diffusion parameter influencing the noise level of the variance $v_{t}$.

It is convenient to change the variable from price $S_{t}$ to the adjusted log-return $x_{t}=\log \left(S_{t} / S_{0}\right)-\mu t$. Using Itô's formula we obtain

$$
d x_{t}=-\frac{v_{t}}{2} d t+\sqrt{v_{t}} d W_{t}^{(1)} .
$$

The two SDE for the variance $v_{t}$ and the adjusted log-return $x_{t}$ define a two-dimensional stochastic process

$$
\left(\begin{array}{c}
x_{t} \\
v_{t}
\end{array}\right)=-\left(\begin{array}{c}
v_{t} / 2 \\
\gamma(v-\theta)
\end{array}\right) d t+\sqrt{v_{t}}\left(\begin{array}{cc}
1 & 0 \\
\kappa \rho & \kappa \sqrt{1-\rho^{2}}
\end{array}\right)\left(\begin{array}{c}
d W_{t}^{(1)} \\
d Z_{t}
\end{array}\right) .
$$

The stochastic process $t \rightarrow\left(x_{t}, v_{t}\right)$ possesses a conditional joint probability density $p\left(x_{t}, v_{t} \mid v_{0}\right)$ with the initial condition $v=v_{0}$ for the variance. The initial condition of the SDE for the adjusted log-return $x_{t}$ is always 0 by definition and therefore not mentioned explicitly. The time evolution of the probability density is governed by the Fokker-Planck equation

$$
\partial_{t} p=\gamma \partial_{v}[(v-\theta) p]+\frac{1}{2} \partial_{x}(v p)+\rho \kappa \partial_{x v}(v p)+\frac{1}{2} \partial_{x x}(v p)+\frac{\kappa^{2}}{2} \partial_{v v}(v p)
$$

with initial condition

$$
p\left(x_{0}, v_{i} \mid v_{0}\right)=\delta\left(x_{0}\right) \delta\left(v_{i}-v_{0}\right)
$$

where $\delta(\cdot)$ denotes the Dirac delta function. The stationary density of $v$ has been computed by Dragulescu and Yakovenko as [15]

$$
\pi_{*}(v)=\frac{\alpha^{\alpha}}{\Gamma(\alpha)} \frac{v^{\alpha-1}}{\theta^{\alpha}} e^{-\alpha v / \theta}, \quad \alpha=\frac{2 \gamma \theta}{\kappa^{2}}
$$

which is the density of a Gamma distribution with shape $\alpha$ and rate $\beta=\alpha / \theta$. It has mean $\theta$ and variance $\theta / \alpha$ and is well defined as long as $\alpha>0$. Since the FokkerPlanck equation is linear, the superposition

$$
p\left(x_{t}, v_{t}\right)=\int p\left(x_{t}, v_{t} \mid v_{0}\right) \pi_{*}\left(v_{0}\right) d v_{0}
$$

of solutions $p\left(x_{t}, v_{t} \mid v_{0}\right)$ with initial values Eq. (2.7) with respect to the stationary density $\pi_{*}$ is a solution as well with initial condition

$$
\begin{aligned}
p\left(x_{0}, v_{i}\right) & =\int p\left(x_{0}, v_{i} \mid v_{0}\right) \pi_{*}\left(v_{0}\right) d v_{0}=\int \delta(x) \delta\left(v_{i}-v_{0}\right) \pi_{*}\left(v_{0}\right) d v_{0} \\
& =\delta(x) \pi_{*}\left(v_{i}\right) .
\end{aligned}
$$

There exists an analytical solution of the Fokker-Planck equation Eq. (2.6) in form of Fourier and inverse Laplace transforms [15]. Applying the Stehfest method of degree 12 [8] to compute the inverse of the Laplace transform yields an efficient algorithm to solve $p\left(x_{t}, v_{t}\right)$ of Eq. (2.6) with initial condition Eq. (2.10). This 
procedure performs magnitudes faster than solving the Fokker-Planck equation itself numerically, a method we tested also in order to validate our numerical findings by two unrelated computational approaches. Besides the joint density $p\left(x_{t}, v_{t}\right)$ we also need the conditional density

$$
p\left(x_{t} \mid v_{0}\right)=\int_{0}^{\infty} d v_{t} p\left(x_{t}, v_{t} \mid v_{0}\right)
$$

which is the marginal of the conditional joint density $p\left(x_{t}, v_{t} \mid v_{0}\right)$, that is, the solution of the Fokker-Planck equation Eq. (2.6) with respect to the initial conditions Eq. (2.7). There is a Fourier transform representation of the conditional density [15]

$$
\begin{aligned}
p\left(x_{t} \mid v_{0}\right)= & \int_{-\infty}^{\infty} \frac{d p_{x}}{2 \pi} \exp \left(\mathrm{i} p_{x} x-\frac{p_{x}^{2}-\mathrm{i} p_{x}}{\Gamma+\Omega \operatorname{coth}(\Omega t / 2)}\right) \\
& \times \exp \left(-\frac{2 \gamma \theta}{\kappa^{2}} \log \left(\cosh \frac{\Omega t}{2}+\frac{\Gamma}{\Omega} \sinh \frac{\Omega t}{2}\right)+\frac{\gamma \Gamma \theta t}{\kappa^{2}}\right)
\end{aligned}
$$

and if we integrate $v_{0}$ over the stationary distribution with density $\pi_{*}$, we obtain the density of the marginal distribution of the returns [15]

$$
\begin{aligned}
p\left(x_{t}\right)= & \int_{-\infty}^{\infty} \frac{d p_{x}}{2 \pi} \\
& \times \exp \left(\mathrm{i} p_{x} x+\frac{\gamma \theta}{\kappa^{2}} \Gamma t-\frac{2 \gamma \theta}{\kappa^{2}} \log \left(\cosh \frac{\Omega t}{2}+\frac{\Omega^{2}-\Gamma^{2}+2 \gamma \Gamma}{2 \gamma \Omega} \sinh \frac{\Omega t}{2}\right)\right),
\end{aligned}
$$

where we introduced the shorthand $\Gamma=\gamma+i \rho \kappa p_{x}$ and the frequency $\Omega=$ $\sqrt{\Gamma^{2}+\kappa^{2}\left(p_{x}^{2}-\mathrm{i} p_{x}\right)}$.

\subsubsection{Exponential OU models}

While the CIR process is plausible, there exist other popular choices to describe the dynamics of the variance $v_{t}$. For example, the exponential OU model [23] models the logarithm of the variance $y_{t}=\log \sigma_{t}^{2}$ as an OU process, i.e.,

$$
d y_{t}=-\alpha\left(y_{t}-\mu\right) d t+\beta d W_{t}^{(2)} .
$$

Under special conditions, the CIR process can be derived from an OU process: if $v_{t}$ is an CIR process then $\sqrt{v_{t}}$ is an OU process provided that $\gamma \theta=\frac{1}{4} \kappa^{2}$.

The OU process is an example of a wide class of stochastic processes, namely Gaussian processes. These have the property that for any finite collections of times $t_{1}, \ldots, t_{n}$, the observations of the process $y_{t_{1}}, \ldots, y_{t_{n}}$ have a multivariate Gaussian distribution. This in turn implies that the process is completely specified by its mean process $\mu_{t}=\mathbb{E}\left[y_{t}\right]$ and the covariance function $k_{t, t^{\prime}}=\mathbb{E}\left[\left(y_{t}-\mu_{t}\right)\left(y_{t^{\prime}}-\mu_{t^{\prime}}\right)\right]$. For the OU process, the stationary mean process is given by $\mu_{t}^{\mathrm{OU}} \equiv \mu$ and the covariance is well known to be $k_{t, t^{\prime}}^{\mathrm{OU}}=\frac{\beta^{2}}{2 \alpha} e^{-\alpha\left|t-t^{\prime}\right|}$. Below, we also consider other Gaussian processes thereby generalizing the class of stochastic volatility models beyond what is usually studied in finance. 


\subsection{Information theory}

The differential entropy $h(X)$ of a continuous random real-variable $X$ with probability measure $F$ is defined as [11]

$$
\begin{aligned}
h(X) & =-\int \log \frac{d F}{d \mu} d F, \\
& =-\int f(x) \log f(x) d x,
\end{aligned}
$$

where the second line follows the standard convention in information theory that the base measure $\mu$ is the Lebesgue measure and $f(x)$ denotes the probability density of $X$ with respect to the Lebesgue measure, i.e., $f=\frac{d F}{d \mu}$. In the following, we adhere to the sloppy notation common in information theory and consider Eq. (2.6) as the definition of differential entropy. Note that the differential entropy is undefined, if this integral diverges.

Since we measure information in bits we use the logarithm with respect to base 2 . Compared to entropy of discrete random variables, differential entropy loses some important properties. First it can become negative, as probability densities are not bounded below one. A simple example provides a normally distributed random variable $X \sim\left(1 / \sqrt{2 \pi \sigma^{2}}\right) \exp \left(-x^{2} / 2 \sigma^{2}\right)$ whose differential entropy is $1 / 2 \log \left(2 \pi e \sigma^{2}\right)$ [11]. Thus, it can be negative for sufficiently small variances $\sigma^{2} \ll 1$, i.e., when the maximum $1 / \sqrt{2 \pi \sigma^{2}} \gg 1$ of the density becomes sufficiently large. Even though it might become negative, the differential entropy, as the entropy of discrete random variables, can be interpreted as a measure of the average uncertainty in the random variable. Second, due to its implicit dependence on the Lebesgue measure as its base measure it is not invariant under change of variables. Nevertheless, the differential entropy behaves nicely under diffeomorphic coordinate changes $\phi: S_{X} \rightarrow$ $\mathbb{R}$ on the support $S_{X}=\{x: f(x)>0\} \subseteq \mathbb{R}$ of the random variable $X$

$$
h(\phi(X))=h(X)+\int f(x) \log \left|\phi^{\prime}(x)\right| d x .
$$

The differential entropy of a set $X_{1}, X_{2}, \ldots, X_{n}$ of random variables with joint density $f\left(x_{1}, x_{2}, \ldots, x_{n}\right)$ is defined as

$$
\begin{aligned}
h\left(X_{1}, X_{2}, \ldots, X_{n}\right)= & -\int f\left(x_{1}, x_{2}, \ldots, x_{n}\right) \\
& \times \log f\left(x_{1}, x_{2}, \ldots, x_{n}\right) d x_{1} d x_{2} \cdots d x_{n} .
\end{aligned}
$$

For every diffeomorphism $\phi: S_{\mathbf{X}} \rightarrow \mathbb{R}^{n}$ on the support $S_{\mathbf{X}}=\left\{\mathbf{x}=\left(x_{1}, x_{2}, \ldots, x_{n}\right)\right.$ : $\left.f\left(x_{1}, x_{2}, \ldots, x_{n}\right)>0\right\}$ of the random variables $\mathbf{X}=\left(X_{1}, X_{2}, \ldots, X_{n}\right)$, we obtain

$$
h(\phi(\mathbf{X}))=h(\mathbf{X})+\int f(\mathbf{x}) \log \left|\operatorname{det} J_{\phi}(\mathbf{x})\right| d \mathbf{x}
$$

where $J_{\phi}(\mathbf{x})$ is the Jacobian of $\phi$. 
If the random variables $X, Y$ have a joint density function $f(x, y)$ and conditional density function $f(x \mid y)$, respectively, we can define the conditional entropy $h(X \mid Y)$ as

$$
h(X \mid Y)=-\int f(x, y) \log f(x \mid y) d x d y .
$$

Since in general $f(x \mid y)=f(x, y) / f(y)$, we can also write

$$
h(X \mid Y)=h(X, Y)-h(Y)
$$

We must be careful if any of the differential entropies are diverging. $h(X \mid Y)$ is a measure of the average uncertainty of the random variable $X$ conditional on the knowledge of another random variable $Y$. The mutual information $I(X: Y)$ between two random variables $X$ and $Y$ is defined as

$$
I(X: Y)=h(X)-h(X \mid Y)=h(Y)-h(Y \mid X) .
$$

Again, we must be careful if any of the differential entropies are infinite and the mutual information might or might not diverge in this case. Mutual information is symmetric, i.e., $I(X: Y)=I(Y: X)$. Further, $I(X: Y) \geq 0$ with equality if and only if $X$ and $Y$ are independent. Thus, mutual information can be considered as a general measure of statistical dependence as it detects any deviations from independence. Note that in the case of independence, knowledge of $X$ does not reduce our uncertainty about $Y$, i.e., $X$ provides no information about $Y$, and vice versa. The mutual information can also be written in terms of the joint probability density $p(x, y)$ of $X$ and $Y$ :

$$
I(X: Y)=\int p(x, y) \log \frac{p(x, y)}{p(x) p(y)} d x d y=\int p(x) p(y \mid x) \frac{p(y \mid x)}{p(y)} d x d y,
$$

where $p(x)$ and $p(y)$ denote the marginal densities of $X$ and $Y$, respectively, and $p(y \mid x)$ denotes the conditional density of $Y$ given $X$. Note that the base measure cancels in this formula and accordingly, in contrast to differential entropy, mutual information behaves as in the discrete case. In particular, it is nonnegative with equality if and only if $X$ and $Y$ are independent and invariant to coordinate changes.

Like mutual information, conditional mutual information $I(X: Y \mid Z)$ between three random variables $X, Y$ and $Z$ can be written in terms of conditional entropies as

$$
I(X: Y \mid Z)=h(X \mid Z)-h(X \mid Y, Z)
$$

assuming the differential entropies exist. This can be rewritten to show its relationship to mutual information

$$
I(X: Y \mid Z)=I(X: Y, Z)-I(X: Z)
$$

usually rearranged as the chain rule of mutual information [11]

$$
I(X: Y, Z)=I(X: Y \mid Z)+I(X: Z) .
$$


In contrast to differential entropy, mutual information and conditional mutual information are scaling invariant, that is, for three diffeomorphic maps $\phi_{X}, \phi_{Y}, \phi_{Z}$ : $\mathbb{R} \rightarrow \mathbb{R}$ we have $[18]$

$$
\begin{aligned}
& I\left(\phi_{X}(X): \phi_{Y}(Y)\right)=I(X: Y) \\
& I\left(\phi_{X}(X): \phi_{Y}(Y) \mid \phi_{Z}(Z)\right)=I(X: Y \mid Z) .
\end{aligned}
$$

\subsection{Multilevel dynamical systems}

We consider the stock-volatility process $t \rightarrow\left(S_{t}, \sigma_{t}\right)$ with a fixed initial value $S_{0}$, the stock price today, and a random volatility $\sigma_{0}$. Since volatility is a hidden parameter, the only observable is the stock $S_{t}$. Then, we discretize the continuous processes $S_{t}$ and $\sigma_{t}$ in time with resolution $\tau$. This is sensible because the observable, that is, stock data, is in general only quoted at discrete time points, most commonly at the end of every trading day, i.e., $\tau=1$ day. From this we obtain a Markov process in discrete time $n \rightarrow\left(S_{n \tau}, \sigma_{n \tau}\right)$. Since only the stock price can be observed, we can illustrate the situation as in Fig. 1 with $\phi$ being the observation map that simply projects onto its first component.

We investigate to which extent the observed process $n \rightarrow S_{n \tau}$ is a stochastic process in its own right. The dashed line on the upper level indicates that the upper process is not self-contained, because $S_{(n+1) \tau}$ cannot in general be derived from $S_{n \tau}$ solely, whereas the solid line on the lower level indicates the Markovian dynamics for the stochastic differentials for $S_{n \tau}$ and $\sigma_{n \tau}$ or, equivalently, $x_{n \tau}$ and $v_{n \tau}$ via Eq. (2.5). Such multilevel dynamical systems were extensively studied by Pfante et al. [27] who introduced various information theoretical measures in order to make the notion "self-contained" precise and the deviation of a process from being self-contained quantifiable. Two of them are of particular interest.

Informational closure: We called the higher process to be informationally closed, if there is no information flow from the lower to the higher level. Knowledge of the joint state $\left(S_{n \tau}, \sigma_{n \tau}\right)$ will not improve predictions of the stock $S_{(n+1) \tau}$ at time $n+1$ when $S_{n \tau}$ is already known, i.e., for $S_{(n+1) \tau}=\phi\left(S_{(n+1) \tau}, \sigma_{(n+1) \tau}\right)$, we have

$$
I\left(S_{(n+1) \tau}:\left(S_{n \tau}, \sigma_{n \tau}\right) \mid S_{n \tau}\right)=0 .
$$

Markovianity: Markovianity of the upper process $n \rightarrow S_{n \tau}$ is another property that allows it to be considered as a self-contained process in its own right. In this case

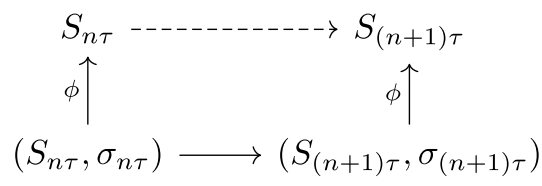

Fig. 1. Basic setup of a low level process that can be observed via an observation map $\phi$. 
$S_{(n+1) \tau}$ is independent of the entire past trajectory

$$
\mathbf{S}_{n-1}^{\tau}=\left(S_{(n-1) \tau}, \ldots, S_{\tau}, S_{0}\right)
$$

given $S_{n \tau}$, which can be expressed again in terms of the conditional mutual information as

$$
I\left(S_{(n+1) \tau}: \mathbf{S}_{n-1}^{\tau} \mid S_{n \tau}\right)=0 .
$$

In our setting, i.e., with $S_{t}$ and $\sigma_{t}$ derived from a SDE as in Sec. 2.1, we obtain Lemma 1. Scaling invariance Eq. (2.27) of the mutual information implies

$$
I\left(S_{(n+1) \tau}:\left(S_{n \tau}, \sigma_{n \tau}\right) \mid S_{n \tau}\right)=I\left(x_{(n+1) \tau}: v_{n \tau} \mid x_{n \tau}\right)
$$

for all $\tau>0$ and $n \in \mathbb{N}$.

Proof. Equation (2.27) implies

$$
I\left(S_{(n+1) \tau}:\left(S_{n \tau}, \sigma_{n \tau}\right) \mid S_{n \tau}\right)=I\left(x_{(n+1) \tau}:\left(x_{n \tau}, v_{n \tau}\right) \mid x_{n \tau}\right) .
$$

Then, by the chain rule of mutual information Eq. (2.26) this can be expanded as

$$
I\left(x_{(n+1) \tau}:\left(x_{n \tau}, v_{n \tau}\right) \mid x_{n \tau}\right)=I\left(x_{(n+1) \tau}: v_{n \tau} \mid x_{n \tau}\right)+I\left(x_{(n+1) \tau}: x_{n \tau} \mid x_{n \tau}, v_{n \tau}\right) .
$$

The result then follows from $I\left(x_{(n+1) \tau}: x_{n \tau} \mid x_{n \tau}, v_{n \tau}\right)=0$.

As a corollary, for the first step, i.e., $n=0$, we obtain

\section{Corollary 1.}

$$
I\left(S_{\tau}:\left(S_{0}, \sigma_{0}\right) \mid S_{0}\right)=I\left(x_{\tau}: v_{0}\right)
$$

from Eq. (2.27) and by dropping the implicit condition $x_{0}=0$.

Similarly, we can derive a bound on the deviation from Markovianity for the stock process $n \rightarrow S_{n \tau}$ based on the information flow.

Theorem 1. In the setting of Fig. 1

$$
I\left(S_{(n+1) \tau}: \mathbf{S}_{n-1}^{\tau} \mid S_{n \tau}\right) \leq I\left(x_{(n+1) \tau}: v_{n \tau} \mid x_{n \tau}\right)
$$

for all $\tau>0$ and $n \in \mathbb{N}$.

Proof. Scale invariance and decomposing the mutual information yields

$$
\begin{aligned}
I\left(S_{(n+1) \tau}: \mathbf{S}_{n-1}^{\tau} \mid S_{n \tau}\right) & =I\left(x_{(n+1) \tau}: \mathbf{x}_{n-1}^{\tau} \mid x_{n \tau}\right) \\
& \leq I\left(x_{(n+1) \tau}:\left(\mathbf{x}_{n-1}^{\tau}, v_{n \tau}\right) \mid x_{n \tau}\right) \\
& =I\left(x_{(n+1) \tau}: v_{n \tau} \mid x_{n \tau}\right)+I\left(x_{(n+1) \tau}: \mathbf{x}_{n-1}^{\tau} \mid x_{n \tau}, v_{n \tau}\right) .
\end{aligned}
$$

Markovianity of the process $n \rightarrow\left(x_{n \tau}, v_{n \tau}\right)$ yields $I\left(x_{(n+1) \tau}: \mathbf{x}_{n-1}^{\tau} \mid x_{n \tau}, v_{n \tau}\right)=0$. 


\section{Results}

\subsection{Heston's model}

As an example for our numerical studies we take realistic parameters from Ait-Sahalia and Kimmel [1] who fitted Heston's model on the S\&P 500 and its VIX. They got the values

$$
\gamma=5.07, \quad \theta=0.0457, \quad \kappa=0.48, \quad \rho=-0.767 .
$$

The parameters Eq. (3.1) are those for an annual time scale: $t=1$ corresponds to one year, i.e., 252 trading days.

\subsubsection{Mutual information between stock price and volatility}

Originally, we computed the mutual information $I\left(v_{t}: x_{t}\right)$ but due to the scaling invariance Eq. (2.27) of mutual information we have $I\left(\sigma_{t}: S_{t}\right)=I\left(v_{t}: x_{t}\right)$. Several remarkable facts can be read off Fig. 2. First, the maximum $I\left(\sigma_{\tau}: S_{\tau}\right)=0.48$ bits at $\tau=55$ trading days. Second, the mutual information diminishes for longer times $t$. Third, and most important, the small amount of information left in the stock about its underlying volatility. We get at most about half a bit which makes at least 25 independent measurements necessary to derive $10 \mathrm{bits}$, that is about three significant digits of the volatility. Fourth, the instantaneous jump of the mutual information $I\left(v_{t}: x_{t}\right)$ for $t \rightarrow 0$. Since the distributions of the adjusted log-return $x_{t}$ and the variance $v_{t}$ are chosen to be independent at $t=0$, as of Eq. (2.10), mutual

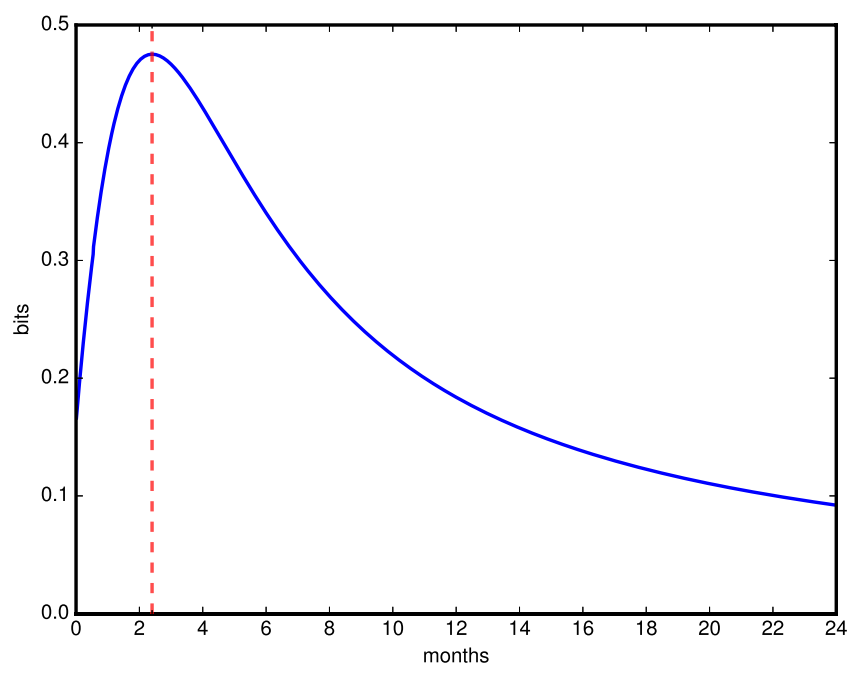

Fig. 2. The evolution of the mutual information $I\left(\sigma_{t}: S_{t}\right)$ for the joint density $p\left(x_{t}, v_{t}\right)$ solving the PDE Eq. (2.6) with initial condition Eq. (2.10). The maximum is approximately at $t=55$ trading days. 
information $I\left(x_{0}: v_{0}\right)=0$. But the numerical simulation suggests the limit $\lim _{t \rightarrow 0} I$ $\left(x_{t}: v_{t}\right) \approx 0.166$ bits.

\subsubsection{Informational flow}

The informational flow of Sec. 2.3 reads

$$
I\left(S_{(n+1) \tau}:\left(S_{n \tau}, \sigma_{n \tau}\right) \mid S_{n \tau}\right)=I\left(S_{(n+1) \tau}: \sigma_{n \tau} \mid S_{n \tau}\right)
$$

Scaling invariance Eq. (2.27) of mutual information yields

$$
\begin{aligned}
I\left(S_{(n+1) \tau}: \sigma_{n \tau} \mid S_{n \tau}\right) & =I\left(x_{(n+1) \tau}: v_{n \tau} \mid x_{n \tau}\right) \\
& =h\left(x_{(n+1) \tau} \mid x_{n \tau}\right)-h\left(x_{(n+1) \tau} \mid x_{n \tau}, v_{n \tau}\right) \\
& =h\left(x_{(n+1) \tau}, x_{n \tau}\right)-h\left(x_{n \tau}\right)-h\left(x_{\tau} \mid x_{0}, v_{0}\right)
\end{aligned}
$$

where the last equality follows from $p\left(x_{(n+1) \tau} \mid x_{n \tau}, v_{n \tau}\right)=p\left(x_{\tau} \mid x_{0}, v_{0}\right)$, i.e., the temporal homogeneity of solutions of the Fokker-Planck equation Eq. (2.6). The conditional density $p\left(x_{\tau} \mid x_{0}, v_{0}\right)$ is in general not equal Eq. (2.12) as long as the condition on $x_{0}$ is replaced by $x_{0}=0$. If $x_{0}=x$, then

$$
\begin{aligned}
h\left(x_{\tau} \mid x_{0}, v_{0}\right) & =-\int p\left(x_{\tau}=y, x_{0}, v\right) \log p\left(x_{\tau}=y \mid x_{0}, v\right) d x_{0} d v d y \\
& =-\int p\left(x_{\tau}=y \mid x_{0}=x, v_{0}\right) \log p\left(x_{\tau}=y \mid x_{0}=x, v_{0}\right) d y
\end{aligned}
$$

where the second identity follows from the fact that $x_{0}$ and $v$ are Dirac distributed at $x$ and $v_{0}$, respectively. If we change the coordinates $y^{\prime}=y-x$ and $x^{\prime}=x$, the Jacobian of this coordinate transformation is 1 and we obtain

$$
h\left(x_{\tau} \mid x_{0}, v_{0}\right)=-\int p\left(x_{\tau}=y^{\prime}+x^{\prime} \mid x_{0}=x^{\prime}, v_{0}\right) \log p\left(x_{\tau}=y^{\prime}+x^{\prime} \mid x_{0}=x^{\prime}, v_{0}\right) d y^{\prime} .
$$

Since solutions $\left(x_{t}, v_{t}, x_{0}, v_{0}\right) \mapsto p\left(x_{t}, v_{t} \mid x_{0}, v_{0}\right)$ of the partial differential equation Eq. (2.6) depend only on $\left(x_{t}-x_{0}, v_{t}, 0, v_{0}\right)$, we obtain $p\left(x_{\tau}=y^{\prime}+x^{\prime} \mid x_{0}=\right.$ $\left.x^{\prime}, v_{0}\right)=p\left(x_{\tau}=y^{\prime} \mid x_{0}=0, v_{0}\right)$. This implies $h\left(x_{\tau} \mid x_{0}, v_{0}\right)=h\left(x_{\tau} \mid v_{0}\right)$ adopting the rule that we drop the implicit condition $x_{0}=0$. Hence, the entropy $h\left(x_{\tau} \mid x_{0}, v_{0}\right)$ is the one of the conditional density Eq. (2.12). Computing the joint density $p\left(x_{(n+1) \tau}, x_{n \tau}\right)$ yields

$$
\begin{aligned}
p\left(x_{(n+1) \tau}, x_{n \tau}\right) & =\int p\left(x_{(n+1) \tau}, x_{n \tau}, v_{n \tau}=v\right) d v \\
& =\int p\left(x_{(n+1) \tau} \mid x_{n \tau}, v_{n \tau}=v\right) p\left(x_{n \tau}, v_{n \tau}=v\right) d v \\
& =\int p\left(x_{\tau} \mid x_{0}, v_{0}=v\right) p\left(x_{n \tau}, v_{n \tau}=v\right) d v
\end{aligned}
$$


Its entropy reads

$$
\begin{aligned}
h\left(x_{(n+1) \tau}, x_{n \tau}\right)= & -\int p\left(x_{(n+1) \tau}=y, x_{n \tau}=x\right) \log p\left(x_{(n+1) \tau}=y, x_{n \tau}=x\right) d x d y \\
= & -\int\left(\int p\left(x_{\tau}=y \mid x_{0}=x, v_{0}=v\right) p\left(x_{n \tau}=x, v_{n \tau}=v\right) d v\right) \\
& \times \log \left(\int p\left(x_{\tau}=y \mid x_{0}=x, v_{0}=v\right) p\left(x_{n \tau}=x, v_{n \tau}=v\right) d v\right) d x d y .
\end{aligned}
$$

Furthermore, changing coordinates $y^{\prime}=y-x$ and $x^{\prime}=x$ yields

$$
\begin{aligned}
h\left(x_{(n+1) \tau}, x_{n \tau}\right)= & -\int\left(\int p\left(x_{\tau}=y^{\prime}+x^{\prime} \mid x_{0}=x^{\prime}, v_{0}=v\right) p\left(x_{n \tau}=x^{\prime}, v_{n \tau}=v\right) d v\right) \\
& \times \log \left(\int p\left(x_{\tau}=y^{\prime}+x^{\prime} \mid x_{0}=x^{\prime}, v_{0}=v\right) p\left(x_{n \tau}=x^{\prime}, v_{n \tau}=v\right) d v\right) d x^{\prime} d y^{\prime} .
\end{aligned}
$$

We $\quad$ substituted $\quad p\left(x_{\tau}=y^{\prime}+x^{\prime} \mid x_{0}=x^{\prime}, v_{0}=v\right)=p\left(x_{\tau}=y^{\prime} \mid x_{0}=0, v_{0}=v\right) \quad$ and reduced Eq. (3.3) to expressions which can be computed via Eq. (2.12), at least numerically. We simulated the informational flow Eq. (2.28) for several choices of $n$.

Besides the informational flow, we are also interested in the mutual information $I\left(S_{(n+1) \tau}: S_{n \tau}\right)=I\left(x_{(n+1) \tau}: x_{n \tau}\right)$ between two subsequent observations of the stock. Definition of the conditional mutual information yields

$$
\begin{aligned}
I\left(x_{(n+1) \tau}: v_{n \tau} \mid x_{n \tau}\right) & =I\left(x_{(n+1) \tau}: v_{n \tau}, x_{n \tau}\right)-I\left(x_{(n+1) \tau}: x_{n \tau}\right) \\
& =h\left(x_{(n+1) \tau}\right)-h\left(x_{(n+1) \tau} \mid v_{n \tau}, x_{n \tau}\right)-I\left(x_{(n+1) \tau}: x_{n \tau}\right) \\
& =h\left(x_{(n+1) \tau}\right)-h\left(x_{\tau} \mid v_{0}, x_{0}\right)-I\left(x_{(n+1) \tau}: x_{n \tau}\right) \\
& =h\left(x_{(n+1) \tau}\right)-h\left(x_{\tau} \mid v_{0}\right)-I\left(x_{(n+1) \tau}: x_{n \tau}\right),
\end{aligned}
$$

where the last equation follows from the previously proven identity $h\left(x_{\tau} \mid v_{0}, x_{0}\right)=$ $h\left(x_{\tau} \mid v_{0}\right)$ and the last entropy is the one of the conditional density Eq. (2.12). The entropy $h\left(x_{(n+1) \tau}\right)$ can be computed via formula Eq. (2.13) for the density of $x_{t}$. Hence,

$$
I\left(S_{(n+1) \tau}: S_{n \tau}\right)=I\left(x_{(n+1) \tau}: x_{n \tau}\right)=h\left(x_{(n+1) \tau}\right)-h\left(x_{\tau} \mid v_{0}\right)-I\left(x_{(n+1) \tau}: v_{n \tau} \mid x_{n \tau}\right) .
$$

In particular, if we set $n=0$, we obtain $I\left(S_{\tau}: S_{0}\right)=0$. For the other values of $n$ we plotted in Fig. 4 the information ratio

$$
I R_{n \tau}=\frac{I\left(S_{(n+1) \tau}: \sigma_{n \tau} \mid S_{n \tau}\right)}{I\left(S_{(n+1) \tau}: S_{n \tau}\right)}
$$

which expresses the informational gain on predictions of future stock movements $S_{(n+1) \tau}$ we obtain from knowing the instantaneous volatility compared to the information already there from reading off stock data $S_{n \tau}$ at time $n \tau$. 


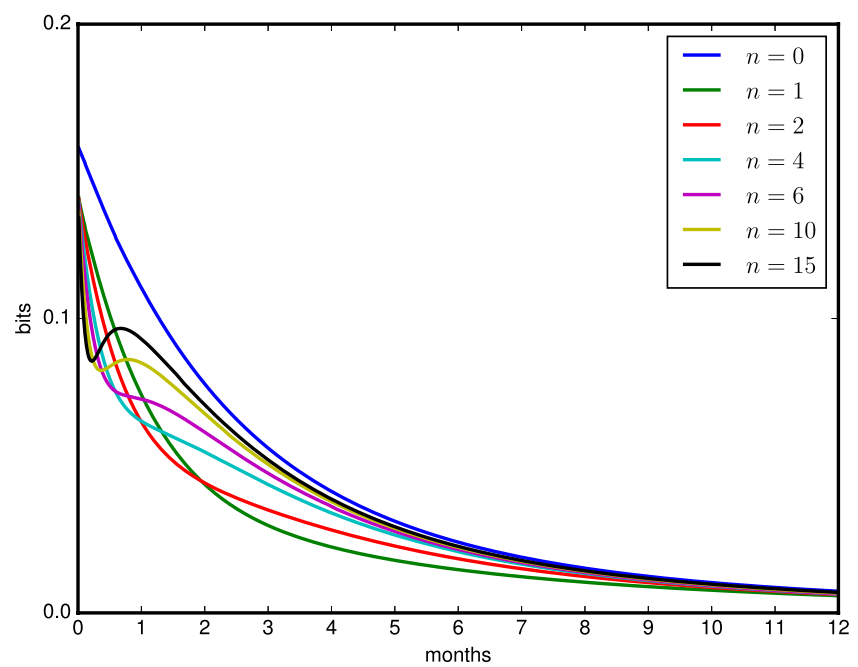

Fig. 3. The informational flow $I\left(S_{(n+1) \tau}: \sigma_{n \tau} \mid S_{n \tau}\right)$ for $n=0,1,2,4,6,10$, and 15 .

Several facts can be read off Figs. 3 and 4 . First, the informational flow $I\left(S_{\tau}\right.$ : $\left.\sigma_{0} \mid S_{0}\right)=I\left(x_{\tau}: v_{0}\right)$ is an upper bound of the general flow $I\left(S_{(n+1) \tau}: \sigma_{n \tau} \mid S_{n \tau}\right)=$ $I\left(x_{(n+1) \tau}: v_{n \tau} \mid x_{n \tau}\right)$ for all $n \in \mathbb{N}_{0}$. Second, the incredible small amount of this flow: the knowledge of the joint state $\left(S_{n \tau}, \sigma_{n \tau}\right)$ will not markably improve predictions on the stock $S_{(n+1) \tau}$ at time $\tau$ if $S_{n \tau}$ is already known. The informational gain $I\left(S_{(n+1) \tau}\right.$ : $\left.\sigma_{n \tau} \mid S_{n \tau}\right)$ compared to the information $I\left(S_{(n+1) \tau}: S_{n \tau}\right)$ we already have about $S_{(n+1) \tau}$ if we know $S_{n \tau}$ is at most $27 \%$ and vanishes if $\tau$ or $n$ increases. Diminishing

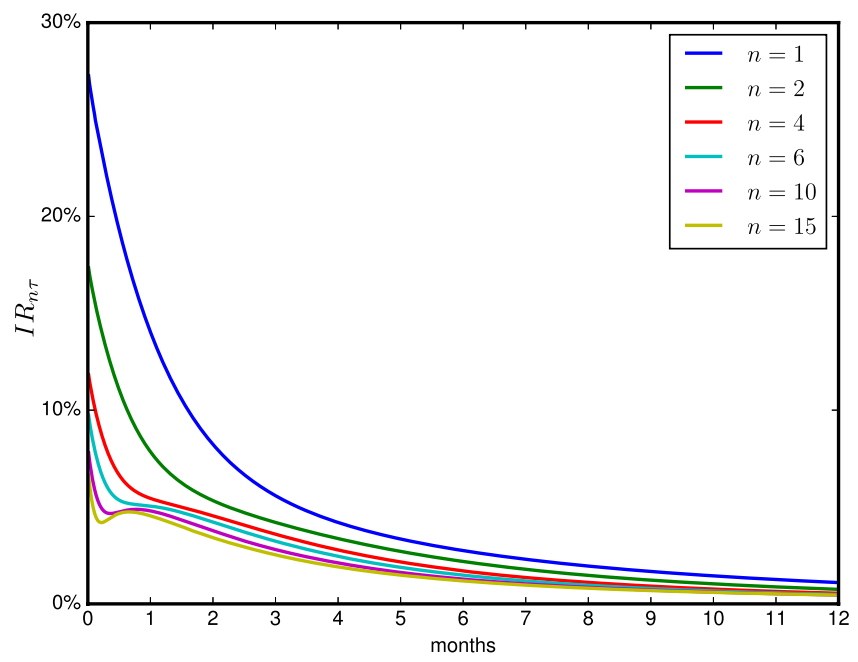

Fig. 4. The information ratio $I R_{n \tau}$ for $n=1,2,4,6,10$, and 15 . 
informational gain on future returns from knowing the instantaneous volatility $v_{n \tau}$ if $n$ grows can be directly derived from Eq. (3.5): we already know from Fig. 3 that $I\left(S_{(n+1) \tau}: \sigma_{n \tau} \mid S_{n \tau}\right)$ is upper bounded by $I\left(S_{\tau}: \sigma_{0} \mid S_{0}\right)=h\left(x_{\tau}\right)-h\left(x_{\tau} \mid v_{0}\right)$ independent of $n$, whereas $h\left(x_{(n+1) \tau}\right)$ is extensive in $n$ because the marginal distribution of $x_{(n+1) \tau}$ is roughly Gaussian with a variance proportional to $(n+1) \tau$. Hence, $h\left(x_{(n+1) \tau}\right) \approx \mathcal{O}(\log ((n+1) \tau))$. The same argument yields $h\left(x_{\tau} \mid v_{0}\right) \approx \mathcal{O}(\log (\tau))$ and therefore

$$
I\left(S_{(n+1) \tau}: S_{n \tau}\right)=I\left(x_{(n+1) \tau}: x_{n \tau}\right) \approx \log ((n+1) \tau)-\log \tau=\log (n+1),
$$

that is, the denominator in Eq. (3.6) grows logarithmically in $n$. Overall, knowing the instantaneous volatility $\sigma_{n \tau}$ does not deliver considerably better estimations on future stock returns than the knowledge of the stationary density $\pi_{*}$ of the volatility and variance, does, which we simply know from the model. Therefore, inferring the realized volatility from market data in financial markets subject to Heston's model is not only hard, but also has a limited effect on the future price process. Similarly, from theorem 1 follows that the stock process $n \rightarrow S_{n \tau}$ is nearly Markovian for increasing $\tau$. Together these observations suggests that especially when pricing options of long maturity modeling of the stationary density of future prices is more important than capturing the volatility dynamics. Indeed, Lévy processes with independent, but non-Gaussian price returns have been proposed as practical alternatives for pricing options and other derivatives [31]. Last, also the informational flow $I\left(S_{(n+1) \tau}: \sigma_{n \tau} \mid S_{n \tau}\right)$ makes an instantaneous jump for $\tau \rightarrow 0$ with the same jump height for $n=0$ as the mutual information $I\left(x_{\tau}: v_{\tau}\right)$ in Fig. 2.

\subsection{Exponential Ornstein-Uhlenbeck models}

\subsubsection{Fitting the models}

The mutual information $I\left(S_{t}: \sigma_{t}\right)$ quantifies the average dependence between the stock price and instantaneous volatility which we have evaluated for the Heston model above. When inferring volatility from actual stock price data we are usually less interested in the average behavior over possible realizations of the price process $t \rightarrow S_{t}$. Rather, we want to estimate the volatility conditioned on the actual sequence of prices $\mathbf{S}=\left(S_{t_{1}}, \ldots, S_{t_{n}}\right)$ that were observed at times $t_{1}<\cdots<t_{n}$. Unfortunately, computing the full conditional density $p\left(\sigma_{t} \mid \mathbf{S}\right)$ or even $p\left(\left\{\sigma_{t}\right\}_{t>0}: \mathbf{S}\right)$ is not analytically feasible in the Heston model. We note that the algorithm by Bates [3] allows to approximate the characteristic function of the former conditional density. Here, instead we take a different approach and turn to a different class of models which is based on Gaussian processes. While exact inference is also infeasible in these models, Gaussian processes are heavily used in machine learning and a wide range of approximation algorithms is readily available, for both of the above conditional densities. As described above, we consider generalizations of the exponential OU model. 
As before, the price process is given by

$$
d S_{t}=\mu S_{t} d t+\sigma_{t} S_{t} d W_{t}^{(1)},
$$

but now, the logarithm of the variance $y_{t}=\log \sigma_{t}^{2}$ is drawn from a Gaussian process. To fit such a model, we can represent the distribution of $\mathbf{y}=\left(y_{t_{1}}, \ldots, y_{t_{n}}\right)$ exactly by a multivariate Gaussian distribution. In addition, we need the likelihood of the observed prices $\mathbf{S}$ given $\mathbf{y}$, i.e., $p(\mathbf{S} \mid \mathbf{y})$. Here, for simplicity, we use an Euler approximation to the SDE for $S_{t}[28]$ :

$$
S_{t+\Delta t}-S_{t}=\mu S_{t} \Delta t+\sigma_{t} S_{t} \sqrt{\Delta t} \epsilon_{t}
$$

where $\epsilon_{t} \sim \mathcal{N}(0,1)$.

Thus, we assume that the observed daily return $r_{t}=\frac{S_{t+1}-S_{t}}{S_{t}}$ is drawn from a normal distribution with standard deviation $\sigma_{t}$, i.e., $r_{t} \sim \mathcal{N}\left(\mu, \sigma_{t}\right)$. Since the drift of the price process is usually rather small compared to its volatility, we will assume $\mu=0$ for simplicity in the following.

In this formulation, our stochastic volatility model is a Gaussian process $y_{t}$ with a non-Gaussian likelihood model $p\left(r_{t} \mid y_{t}\right)$. Similar models are well known and widely used in Gaussian process classification [29, Chap. 3]. In this context, several algorithms to approximate the posterior $p(\mathbf{y} \mid \mathbf{r})$ have been developed. Here, we employ the Laplace approximation where the posterior is approximated by a multivariate Gaussian centered at the mode of the posterior, i.e., $\mathbf{y}_{\text {Laplace }}=\operatorname{argmax}_{\mathbf{y}} p(\mathbf{y} \mid \mathbf{r})=$ $\operatorname{argmax}_{\mathbf{y}} \Psi(\mathbf{y})$, where $\Psi(\mathbf{y})=\log p(\mathbf{r} \mid \mathbf{y}) p(\mathbf{y})$. The covariance for the approximate posterior is derived from the local curvature around the mode, i.e., $\Sigma_{\text {Laplace }}^{-1}=$ $-\nabla \nabla \log p\left(\mathbf{y}_{\text {Laplace }} \mid \mathbf{r}\right)$.

Using the Laplace approximation it is also possible to compute an approximation to the marginal likelihood

$$
\begin{aligned}
p(\mathbf{r}) & =\int p(\mathbf{r} \mid \mathbf{y}) p(\mathbf{y}) d \mathbf{y} \\
& \approx e^{\Psi\left(\mathbf{y}_{\text {Laplace }}\right)} \int e^{-\frac{1}{2}\left(\mathbf{y}-\mathbf{y}_{\text {Laplace }}\right)^{T} \Sigma_{\text {Laplace }}^{-1}\left(\mathbf{y}-\mathbf{y}_{\text {Laplace }}\right)} d \mathbf{y} \\
& =p\left(\mathbf{r} \mid \mathbf{y}_{\text {Laplace }}\right) p\left(\mathbf{y}_{\text {Laplace }}\right) \int e^{-\frac{1}{2}\left(\mathbf{y}-\mathbf{y}_{\text {Laplace }}\right)^{T} \Sigma_{\text {Laplace }}^{-1}\left(\mathbf{y}-\mathbf{y}_{\text {Laplace }}\right)} d \mathbf{y} .
\end{aligned}
$$

In contrast to a maximum likelihood solution, $p(\mathbf{r})$ does not just depend on the goodness of fit, as captured by the likelihood $p\left(\mathbf{r} \mid \mathbf{y}_{\text {Laplace }}\right)$. It also takes into account the prior probability $p\left(\mathbf{y}_{\text {Laplace }}\right)$ as well as the uncertainty about the inferred volatility process $\mathbf{y}$. Thus, the marginal likelihood, automatically incorporates a trade-off between model fit and model complexity (nicely explained by MacKay [21, Chap. 28]). For this reason, in Bayesian statistics, the marginal likelihood is often used in model selection.

Here, we use the marginal likelihood to compare different structural assumptions about the underlying volatility process. In the standard exponential stochastic volatility model $y_{t}$ is modelled as an OU process with covariance function 
Table 1. Gaussian processes that are popular in machine learning.

\begin{tabular}{lll}
\hline Name & Covariance & \multicolumn{1}{c}{ Properties } \\
\hline Bias & 1 & constant sample paths \\
Squared Exponential & $e^{-\frac{\left(t-t^{\prime}\right)^{2}}{l^{2}}}$ & infinitely differentiable sample paths \\
Rational Quadratic & $\left(1+\frac{\left(t-t^{\prime}\right)^{2}}{2 \alpha l^{2}}\right)^{-\alpha}$ & infinite mixture of lengthscales \\
\hline
\end{tabular}

$k_{t, t^{\prime}}^{\mathrm{OU}}=\frac{\beta^{2}}{2 \alpha} e^{-\alpha\left|t-t^{\prime}\right|}$. Here, $\frac{1}{\alpha}$ can be considered as a lengthscale as it gives the correlation time of the process. The OU process has continuous, but nowhere differentiable sample paths. In machine learning rather smooth Gaussian processes with differentiable sample paths are usually preferred. Table 1 gives some examples of popular Gaussian processes and their covariance functions.

More complex processes, combining the properties of different processes, can then for example be obtained by adding the corresponding covariance functions. In all our experiments below, we have added a bias covariance function to increase the probability to draw sample paths which are shifted away from zero. This allows our models to easily represent processes $y_{t}$ with a nonzero mean. Overall, we fit and compare processes based on the following covariance structures:

\begin{tabular}{ll}
\hline OU & Bias + Ornstein-Uhlenbeck \\
RBF & Bias + Squared exponential \\
RatQuad & Bias + Rational quadratic \\
RBF_RBF & Bias + Squared exponential + Squared exponential \\
OU-OU & Bias + Ornstein-Uhlenbeck + Ornstein-Uhlenbeck \\
\hline
\end{tabular}

The model OU is just the standard exponential OU model [23], while OU-OU denotes a two-factor version of this model incorporating two independent volatility factors with different time scales. Similarly, the RBF_RBF model is a two-factor extension of the model RBF.

To fit the different models we have then optimized the kernel parameters, e.g., the lengthscale of the OU process, in order to maximize the marginal likelihood of the observed returns. Optimizations have been carried out using the Gaussian process toolbox GPy [16] with gradient descent using the Broyden-Fletcher-Goldfarb-Shanno (BFGS) algorithm from 100 different initial conditions. Using a range of different stock market data (all downloaded from Yahoo finance) we obtain results as shown in Table 2.

Our data sets cover very different time periods as well as sectors and no consistently best model can be identified. Nevertheless, we see a preference for models including two different time scales as either RBF_RBF or OU_OU performs best, closely followed by the RatQuad kernel. The later model exhibits a power-law decay of volatility correlations which is considered as a stylized fact of stock market data. It remains to be investigated why we cannot conform this finding. Nevertheless, also the best fitting two-factor models have a long time scale implying that volatility is correlated over several weeks. In this regard our findings agree with previous results 
Table 2. Results of model comparison on different stock market data sets.

\begin{tabular}{|c|c|c|c|}
\hline Description & Date range & Model & Log. marginal likelihood \\
\hline Apple Inc. & $01 / 01 / 2000$ to $01 / 01 / 2003$ & $\begin{array}{l}\text { RBF_RBF } \\
\text { RatQuad } \\
\text { OU_OU } \\
\text { OU } \\
\text { RBF }\end{array}$ & $\begin{array}{l}1423.22 \\
1421.33 \\
1412.74 \\
1412.23 \\
1403.32\end{array}$ \\
\hline DAX & $01 / 01 / 1992$ to $01 / 01 / 1995$ & $\begin{array}{l}\text { OU_OU } \\
\text { RBF_RBF } \\
\text { RatQuad } \\
\text { RBF } \\
\text { OU }\end{array}$ & $\begin{array}{l}2509.23 \\
2505.98 \\
2498.64 \\
2493.47 \\
2486.51\end{array}$ \\
\hline Exxon Mobile & $01 / 01 / 1986$ to $01 / 01 / 1990$ & $\begin{array}{l}\text { RBF_RBF } \\
\text { OU_OU } \\
\text { RatQuad } \\
\text { OU } \\
\text { RBF }\end{array}$ & $\begin{array}{l}2923.20 \\
2921.43 \\
2906.43 \\
2897.08 \\
2881.11\end{array}$ \\
\hline $\mathrm{S} \& \mathrm{P} 500$ & $01 / 01 / 2006$ to $01 / 01 / 2010$ & $\begin{array}{l}\text { RBF_RBF } \\
\text { OU_OU } \\
\text { RatQuad } \\
\text { OU } \\
\text { RBF }\end{array}$ & $\begin{array}{l}3129.78 \\
3129.58 \\
3093.42 \\
3083.78 \\
3072.75\end{array}$ \\
\hline
\end{tabular}

of econometric studies which strongly suggest the use of two-factor models. In this context, Chernov et al. [9] notes that models "breaking the link between tail thickness and volatility persistence" are needed to fit empirical data.

As an example, Fig. 5 shows the hidden volatility implied by the OU based as well as the best model (RBF_RBF) on the S\&P 500 data. The shaded region shows the $95 \%$ region ( \pm 2 standard deviations from the mean) of the posterior and demonstrates the large uncertainty that remains about the volatility. When predicting the volatility for the next 100 days (red curve), the uncertainty is even more pronounced and increases quickly towards the a priori uncertainty of the stationary volatility distribution. Note that this uncertainty is not due to parameter uncertainty which have been fixed after fitting. The situation is the same as in the Heston model where we also assumed the parameters known and the high uncertainty is due to the stochasticity of the underlying volatility process. It thus appears to be an intrinsic property of stochastic volatility models, especially if the volatility process varies quickly as in the case of realistic parameters.

\subsubsection{Information gain}

As before, we can use information theory to quantify the amount of information obtained about the volatility. While the mutual information quantifies the average uncertainty, here we are interested in the information about $\sigma$ that was obtained from the actually observed prices. DeWeese and Meister [13] propose the difference in entropy between the prior $p(\sigma)$ and the posterior $p(\sigma \mid \mathbf{S})$ as the information gain from 

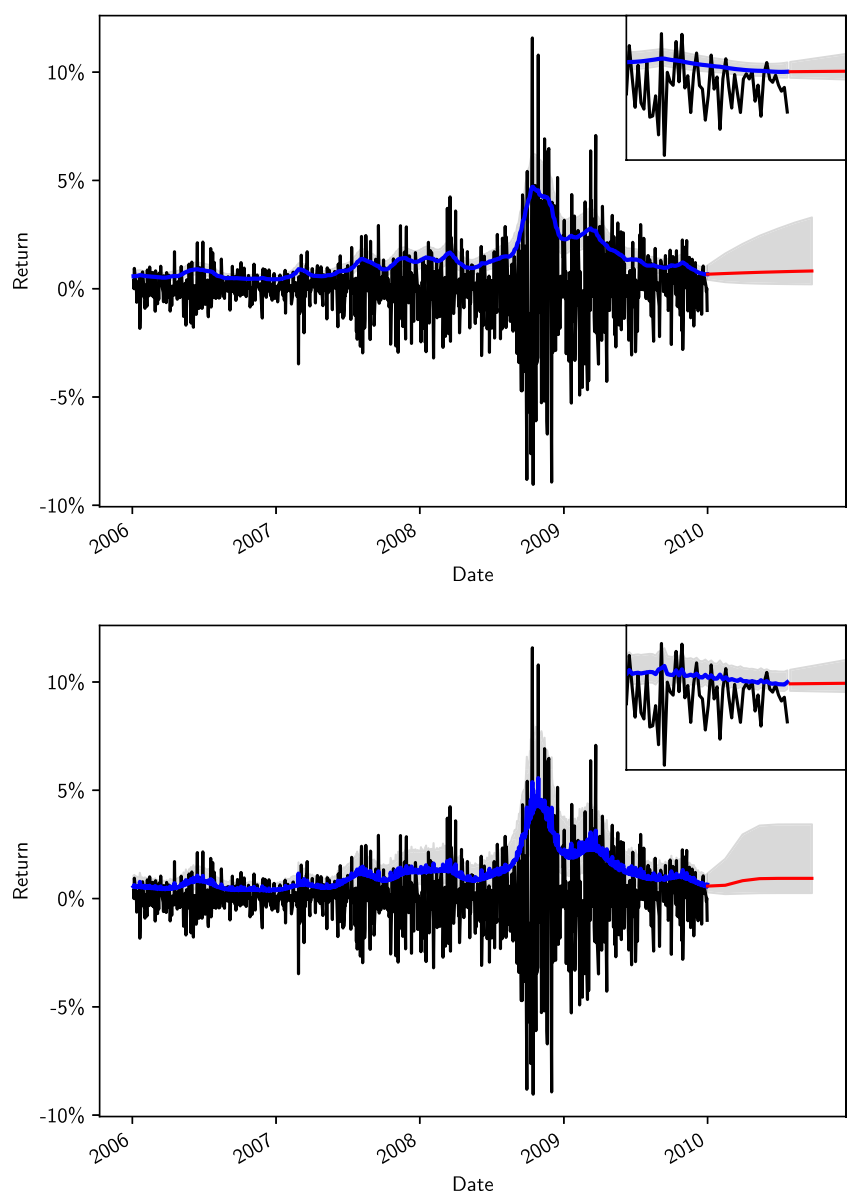

Fig. 5. (Color online) Exponential stochastic volatility model with OU (top) and RBF_RBF (bottom) covariance function fitted to the S\&P 500 data. The daily returns are shown in black with the inferred volatility in blue. The gray region shows the large uncertainty (95\% credibility region) which increases quickly when predicting into the future (red curve). The inset shows a zoom on the last 50 data points.

single observations, i.e., the actual prices $\mathbf{S}$ in our case. Thus, instead of averaging over all possible price realizations as in the conditional entropy $h(\sigma \mid \mathbf{S})$, one computes the entropy of the posterior distribution conditioned on the actually observed prices, i.e., $-\int p(\sigma \mid \mathbf{S}) \log p(\sigma \mid \mathbf{S}) d \sigma$. In our case, these entropies can be evaluated since the posterior density $p(\mathbf{y} \mid \mathbf{S})$ is approximated by a Gaussian and the prior $p(\mathbf{y})$ was assumed to be a Gaussian in the first place. For a $d$-dimensional multivariate Gaussian with covariance matrix $\Sigma$ the differential entropy is given by $\frac{1}{2} \log (2 \pi e)^{d}|\operatorname{det} \Sigma|$. To have a fair comparison with the results obtained for the Heston model, we have taken care that the uncertainty about the mean level of the volatility process did not contribute to the information gain. Thus, as in the Heston model, we assumed that the parameter specifying the stationary mean of the 

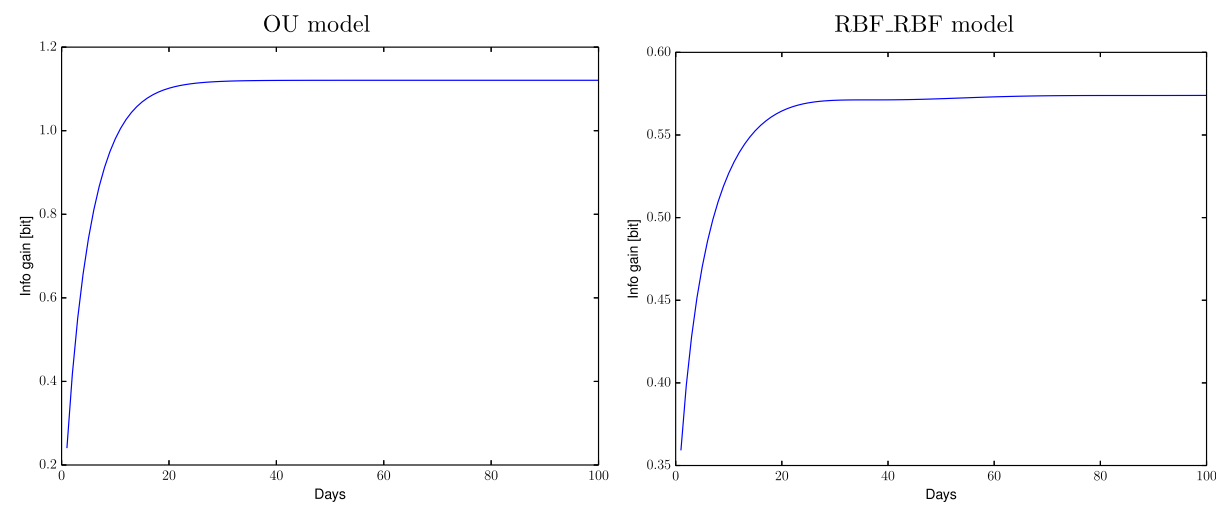

Fig. 6. Information gain about the instantaneous volatility $\sigma_{t}(t=1006)$ based on successively longer sequences of preceding daily returns $r_{t-\tau+1}, \ldots, r_{t}$. In both models the information gain saturates after about 20 days.

volatility process is known. Formally, we accomplish this by removing the bias component of the covariance function before computing the information gain. Note that the entropy of a Gaussian distribution does not depend on the mean of the distribution, and thus adjusting the mean of the prior distribution to its stationary value is not necessary when computing the information gain.

In the example shown in Fig. 5 fitted on 4 years of data (1006 trading days) in total $64.5(\mathrm{OU})$ and 251 bits $\left(\mathrm{RBF} \_\mathrm{RBF}\right)$ are obtained about the volatility time series $\sigma$. The large difference reflects the fact that the OU model is less flexible and thus its a priori uncertainty is already much smaller. Further, the results are consistent with the low information gain found for the Heston model as the above numbers correspond to just 0.06 (OU), respectively, 0.25 (RBF_RBF) bits per observation. While this is comparable to the low values in the Heston model, especially when assuming $\rho=0$, the information about each single observation can well be larger due to the autocorrelation of the volatility process. To illustrate this effect, Fig. 6 shows the information gained about the instantaneous volatility $\sigma_{1006}$, corresponding to the last trading day in our data set, when observing the daily returns preceding it. One clearly sees that observing successive returns does reduce the uncertainty about the volatility, but after just about 20 days the information gain saturates. Thus, even observing years of data would not improve volatility estimates and just about 1 bit of information can be obtained. Again, illustrating the inherent information-theoretic structure of stochastic volatility models, reflected in the restricted range of dependence between observed returns and latent volatility.

\section{Conclusions}

We analyzed Heston's model and exponential OU models from an information theoretic perspective. In particular, we have asked how much information about the 
hidden volatility can be obtained from observing stock prices. First of all, the information of stocks about the volatility which is driving them in the Heston model is 0.5 bit for realistic parameters taken from the S\&P 500. Second, mutual information $I\left(\sigma_{t}, S_{t}\right)$ seems to vanish in the long run or becomes at least meagre. Hence, the processes appear to be almost independent even though the adjusted log-return process $t \rightarrow x_{t}$ is entirely driven by its variance $v_{t}$, see Eq. (2.4). Third, it is not only difficult to infer the instantaneous volatility from stock data, it is of little help regarding stock return predictions: Figs. 3 and 4 show that knowledge of the instantaneous volatility $\sigma_{n \tau}$ improves the prediction of $S_{(n+1) \tau}$ by just a few percent, given that $S_{n \tau}$ is already known. Regarding our results in the third section, this implies that the stock process $n \rightarrow S_{n \tau}$ is close to a Markovian process in its own right.

We find a similar behavior for the exponential stochastic volatility models which we fitted to actual stock price data. Again, we observe a large uncertainty when volatility is inferred from market prices.

\section{Acknowledgment}

This work was supported by the European Research Council under the European Union's Seventh Framework Programme (FP7/2007-2013)/ERC grant agreement No. 318723. Nils Bertschinger thanks Dr. h.c. Maucher for funding his position.

\section{References}

[1] Ait-Sahalia, Y. and Kimmel, R., Maximum likelihood estimation of stochastic volatility models, J. Financial Econom. 83 (2007) 413-452.

[2] Alizadeh, S., Brandt, M. W. and Diebold, F. X., Range-based estimation of stochastic volatility models, J. Finance 57 (2002) 1047-1091.

[3] Bates, D. S., Maximum likelihood estimation of latent affine processes, Rev. Financial Stud. 19 (2006) 909-965.

[4] Black, F. and Scholes, M., The pricing of options and corporate liabilities, J. Political Econom. 81 (1973) 637-654.

[5] Bollerslev, T., Litvinova, J. and Tauchen, G., Leverage and volatility feedback effects in high-frequency data, J. Financial Econom. 4 (2006) 353-384.

[6] Bouchaud, J. P., Matacz, A. and Potters, M., Leverage effect in financial markets: The retarded volatility model, Phys. Rev. Lett. 87 (2001) 228701.

[7] Bouchaud, J.-P. and Potters, M., Theory of Financial Risk and Derivative Pricing, 2nd edn. (Cambridge University Press, Cambridge, England, 2003).

[8] Cheng, A. and Sidauruk, P., Approximate inversion of the Laplace transform, Math. J. 4 (1994) 76-82.

[9] Chernov, M., Gallant, A. R., Ghysels, E. and Tauchen, G., Alternative models for stock price dynamics, J. Econom. 116 (2003) 225-257.

[10] Christie, A. A., The stochastic behavior of common stock variances: Value, leverage and interest rate effects, J. Financial Econom. 10 (1982) 407-432.

[11] Cover, T. M. and Thomas, J. A., Elements of Information Theory, 2nd edn. (WileyInterscience, Hoboken, New Jersey, 2006). 
[12] Cui, Y., del Bao Rollin, S. and Germano, G., Full and fast calibration of the Heston stochastic volatility model, European J. Oper. Res. 263 (2017) 625-638.

[13] DeWeese, M. and Meister, M., How to measure the information gained from one symbol, Network: Comput. Neural Syst. 10 (1999) 325-340.

[14] Ding, Z., Granger, C. W., and Engle, R. F., A long memory property of stock market returns and a new model, J. Empirical Finance 1 (1993) 83-106.

[15] Dragulescu, A. and Yakovenko, V. M., Probability distribution of returns in the Heston model with stochastic volatility, Quant. Finance 2 (2002) 443-453.

[16] GPy, GPy: A Gaussian process framework in python (2012), Available at http://github. com/SheffieldML/GPy.

[17] Heston, S. L., A closed-form solution for options with stochastic volatility with applications to bond and currency options, Rev. Financial Stud. 6 (1993) 327-343.

[18] Kraskov, A., Stögbauer, H. and Grassberger, P., Estimating mutual information, Phys. Rev. E 69 (2004) 066138.

[19] LeBaron, B., Stochastic volatility as a simple generator of apparent financial power laws and long memory, Quant. Finance 6 (2001) 621-631.

[20] Lo, A. W., Long-term memory in stock market prices, Econometrica 59 (1991) $1279-1314$.

[21] MacKay, D. J. C., Information Theory, Inference and Learning Algorithms (Cambridge University Press, Cambridge, England, 2003).

[22] Mandelbrot, B. B., The variation of certain speculative prices, in Fractals and Scaling in Finance: Discontinuity, Concentration, Risk. Selecta Volume E, (Springer, New York, 1997), pp. 371-418.

[23] Masoliver, J. and Perello, J., Multiple time scales and the exponential Ornstein-Uhlenbeck stochastic volatility model, Quant. Finance 6 (2005) 423-433.

[24] Muzy, J. F., Delour, J. and Bacry, E., Modelling fluctuations of financial time series: From cascade process to stochastic volatility model, Eur. Phys. J. B 17 (2000) 537-548.

[25] Pacati, C., Pompa, G. and Renó, R., Smiling twice: The Heston++ model, Available at SSRN: https://ssrn.com/abstract=2697179.

[26] Perello, J., Sircar, R. and Masoliver, J., Option pricing under stochastic volatility: The exponential Ornstein-Uhlenbeck model, J. Statist. Mech. 6 (2008) P06010.

[27] Pfante, O., Bertschinger, N., Olbrich, E., Ay, N. and Jost, J., Comparison between different methods of level identification, Adv. Complex Syst. 17 (2014) 1450007.

[28] Phillips, P. C. B. and Yu, J., Maximum likelihood and Gaussian estimation of continuous time models in finance, in Handbook of Financial Time Series, Mikosch, T., Kreiß, J.-P., Davis, R. A., and Andersen, T. G. eds. (Springer, Berlin Heidelberg, Germany, 2009), pp. $497-530$.

[29] Rasmussen, C. and Williams, C., Gaussian processes for machine learning, in Adaptive Computation and Machine Learning (MIT Press, Cambridge, Massachusetts, 2006).

[30] Rouah, F. D., The Heston Model and its Extensions in Matlab and C\#, 1st edn. (Wiley, Hoboken, New Jersey, 2013).

[31] Schoutens, W., Levy Processes in Finance: Pricing Financial Derivatives, 1st edn. (Wiley, Chichester, England, 2003).

[32] Shannon, C., A mathematical theory of communication, Bell. Syst. Tech. J. 27 (1948) 379-423, 623-656. 